\title{
Mild foot electrical stimulation is comparable with phenytoin in inhibiting pentylenetetrazol-induced kindling in rats
}

\author{
Arefe Ghasemi-Dehno ${ }^{1}$ - Abolfazl Jand ${ }^{1} \cdot$ Monir Abasi-Moghadam ${ }^{1} \cdot$ Mehdi Sadegh $^{1}$ . \\ Morteza Mousavi-Hasanzadeh ${ }^{1} \cdot$ Mohammad Reza Palizvan $^{1}$ (i)
}

Received: 5 May 2019 / Accepted: 28 October 2019 / Published online: 5 November 2019

(c) The Physiological Society of Japan and Springer Japan KK, part of Springer Nature 2019

\begin{abstract}
Increasing evidence demonstrates that electric stimulation has anticonvulsant effects. The present study was undertaken to investigate the effects of mild foot electrical stimulation (MFES) on the development of pentylenetetrazol (PTZ) kindling and compare its effectiveness with the more commonly used treatment, phenytoin. Kindling was induced in rats by repeated injections (every $24 \mathrm{~h}$ ) of PTZ $(37.5 \mathrm{mg} / \mathrm{kg})$. The rats were subjected to either MFES $(0.2 \mathrm{~mA}$ in intensity for a $160 \mathrm{~ms}$ duration with a $160 \mathrm{~ms}$ interval for $20 \mathrm{~min})$ or phenytoin $(30 \mathrm{mg} / \mathrm{kg})$ before PTZ injections. Following this treatment, rats received MFES every other day for 10 days or 26 days after establishment of PTZ kindling. The data showed that MFES significantly inhibited development of chemical kindling induced by PTZ in rats $(p=0.001$, as compared to PTZ-treated animals). This inhibitory effect is comparable with the effect of $30 \mathrm{mg} / \mathrm{kg}$ doses of phenytoin $(P=0.99$, as compared to phenytoin group). However, 10 days or 26 days durations of MFES had no effect on established kindled seizures $(P=0.58$ as compared to PTZ-treated animals). Our data demonstrate that although MFES significantly inhibited the development of chemical kindling, this experimental paradigm had no effect on established kindled seizures.
\end{abstract}

Keywords Pentylenetetrazol $\cdot$ Kindling $\cdot$ Phenytoin $\cdot$ Electrical stimulation $\cdot$ Epilepsy

\section{Introduction}

Epilepsy is a common neurological condition [1]. This disease, if untreated, can cause permanent cerebral complications, such as cognitive impairments [2], learning disabilities [3], intellectual disability, autism spectrum disorders and attention deficit hyperactivity disorder [4, 5]. Epidemiological investigations have shown that over $30 \%$ of epileptic patients experience learning difficulties [6]. Currently, the mainstay for treatment of chronic and recurrent seizures, which is a key symptom of epilepsy, is the development of drugs such as phenytoin and sodium valproate. These drugs control the excitability of neurons in the brain [3].

It has been shown that antiepileptic drugs such as phenytoin have cognitive and behavioral side effects [7]. Furthermore, about $30 \%$ of epileptic patients do not respond to these

Mohammad Reza Palizvan

palizvan@yahoo.com; dr.palizvan@arakmu.ac.ir

1 Department of Physiology, Faculty of Medicine, Arak University of Medical Sciences, Khonin Shahr Street, Sardasht, Arak 38481-7-6941, Iran drugs [8]. This is why it is becoming increasingly important to find new methods for controlling seizures.

Pentylenetetrazol (PTZ) is a selective blocker of the gamma-aminobutyric acid A (GABAA) receptor. It acts on receptors coupled to chloride channels [9]. PTZ was used to induce chemical kindling as an animal model of epilepsy. Such modeling is often used for studying human epilepsy and refers to a phenomenon in which repeated injections of PTZ causes gradual seizure development, culminating in generalized tonic-clonic seizures. There are two phases in the PTZ-induced kindling process. The first phase is epileptogenesis, during which repeated administrations of PTZ culminate with progressive convulsant activity. The second phase is the established epileptic state, referring to the point at which stage five seizures are considered established [10].

There have been many experimental and clinical attempts to control epileptic seizures by using electrical stimulation. Electrical stimulation for the treatment of epilepsy has been proposed in a variety of modalities, such as vagus nerve stimulation [11], deep brain stimulation applied to the anterior nucleus of the thalamus, cerebellum and subthalamic nucleus [12] and spinal cord stimulation [13]. The goal of 
these methods is to provide a treatment with maximum efficacy and minimum adverse effects. Recent studies reported that electrical stimulation can be applied to the skin of the foot and that this low intensity electrical stimulation has beneficial effects on learning and memory performance [14]. However, the effect of mild foot electrical stimulation (MFES) on the development of PTZ kindling and on fully kindled animals has not yet been studied. Therefore, the aim of this study was to investigate the effect of MFES on kindling induced by PTZ and compare it with phenytoin.

\section{Materials and methods}

\section{Animals}

Sixty male Wistar rats weighing 200-250 g each were divided into seven treatment groups. Group $1(n=12)$ received PTZ (37.5 mg/kg, ip injection every second day). In this group 60 min before PTZ injection, each rat was placed in the grid box without electrical stimulation for $20 \mathrm{~min}$. Group $2(n=12)$ received MFES during the kindling process (60 min before PTZ injections). Group $3(n=12)$ received phenytoin during the kindling process ( 30 min before PTZ injections). Group $4(n=6)$ received MFES for 10 days after they reached to the stage five seizure. Group $5(n=6)$ received electric stimulation for 26 days after they reached to the stage five seizure. Group $6(n=6)$ received foot electrical stimulation and group $7(n=6)$ received $30 \mathrm{mg} / \mathrm{kg}$ phenytoin every other day. The rats were housed in environmentally controlled conditions (12 h light-dark cycles, 7:00-19:00 light and 19:00-7:00 dark, temperature $22 \pm 2{ }^{\circ} \mathrm{C}$ ) at the Arak University of Medical Sciences animal facility. Food and water were supplied ad libitum. All procedures were carried out in accordance with EU Directive 2010/63/EU and the University Ethics Committee standards (Arak University of Medical Sciences Research Ethics Committee, ethical approval \# 94-132).

\section{Foot electrical stimulation}

Based on previous studies that reported beneficial effects of low-intensity electrical stimulation on learning and memory [14], the same electrical stimulation parameters were used in this study. Electrical stimulation was induced using a box ( $30 \mathrm{~cm} \times 30 \mathrm{~cm} \times 40 \mathrm{~cm}$ high) with a steel-rod floor (29 parallel rods, $0.3 \mathrm{~cm}$ in diameter, set $1.0 \mathrm{~cm}$ apart). Foot shock consisted electrical stimuli of $0.2 \mathrm{~mA}$ intensity for a $160 \mathrm{~ms}$ duration with a $160 \mathrm{~ms}$ interval applied for $20 \mathrm{~min}$. The stimulation was produced by a constant-current stimulator (WPI, USA) and was delivered through the floor grid.

\section{Kindling}

For induction of kindling, a subconvulsive dose of PTZ $(37.5 \mathrm{mg} / \mathrm{kg}$, ip injection Sigma, USA) was administered every other day for a period of 26 days (13 injections). After each PTZ injection, animals were kept in a Plexiglas chamber $(30 \mathrm{~cm} \times 30 \mathrm{~cm} \times 30 \mathrm{~cm})$ and convulsive behavior was recorded for $30 \mathrm{~min}$. Convulsive responses were classified as described previously [15]. Their parameters were as follows: stage zero, no response; stage one, ear and facial twitching; stage two, myoclonic jerks without upright position; stage three, myoclonic jerks, upright position with bilateral forelimb clonus; stage four, tonic-clonic seizures; and stage five, generalized tonic-clonic seizures, loss of postural control [16]. Rats were considered fully kindled when seizure attacks (stage five) occurred after each PTZ injection for three consecutive injections. The recording parameters were as follows: seizure stage, latency to the onset of stage two and stage five seizures and duration of stage five.

\section{Corticosterone measurement}

Animals were killed by decapitation $1 \mathrm{~h}$ after the last behavioral testing, always occurring between $3 \mathrm{pm}$ and $4 \mathrm{pm}$. Blood was collected and serum corticosterone levels were evaluated. Plasma corticosterone was measured by commercial RIA kits (DRG-Germany). The radioimmunoassay was performed according to the manufacturer's instruction.

\section{Statistical analyses}

Statistical analyses were performed using SPSS Statistics (Version 20). A one-way and two-way analysis of variance (repeated measures and completely randomized) was conducted, followed by a Tukey's test for multiple comparisons. An unpaired Student's $t$ test (two-tailed) was used to compare two different groups of animals. Seizure stage data was analyzed by nonparametric Kruskal-Wallis with post hoc Mann-Whitney $U$ tests. The criterion for statistical significance was $p<0.05$. Results are reported as mean \pm standard deviation of the mean (SD).

\section{Results}

\section{PTZ kindling}

The repeated administration of PTZ $(37.5 \mathrm{mg} / \mathrm{kg}$ ip) produced chemical kindling, as indicated by the progression in seizure stage of PTZ-treated animals. After an average of 13 injections, rats were fully kindled (i.e., 
displayed at least three consecutive stage five seizures) $[F(1,13)=419.71 ; p<0.001$, one-way repeated measure ANOVA) (Fig. 1), and generalized tonic-clonic seizures occurred in all animals.

\section{Effect of pretreatment with phenytoin on the development of PTZ kindling}

The steady increase in seizure stage was markedly inhibited by phenytoin pretreatment $[p<0.001$, time effect $F(12,286)=4.327, p<0.001$; group effect $F(1$, $286)=425.6, p<0.001]$ and time-group interaction $[\mathrm{F}(12$, $286)=2.727, p<0.010$ ] (Fig. 1), and only one rat in this group had stage five seizures. Administration of phenytoin $(30 \mathrm{mg} / \mathrm{kg})$ increased the latency of the onset of stage two seizures. Although one-way ANOVA revealed significant differences among phenytoin, PTZ and PTZ + MFES groups $[\mathrm{F}(4,45)=3.75 ; p<0.010]$, the difference between the phenytoin + PTZ group and the PTZ group was not significant ( $p=0.118$ ) (Fig. 2). The effect of phenytoin pretreatment on stage five seizures has been shown in Fig. 3 . The latency to the onset of stage five seizures significantly increased in the phenytoin-treated rats compared with PTZ rats $[F(4,46)=31.07 ; p=0.001$, one way ANOVA $]$. Post hoc analysis revealed that this difference was significant $(p<0.001)$ as compared to PTZ group. Analyzing the effect of phenytoin on stage five duration showed that pretreatment with phenytoin significantly reduced the stage five duration $[F(4,46)=5.501 ; p=0.001$, one way ANOVA]. Post hoc analysis revealed that this difference was significant for $(p=0.001)$ as compared to PTZ group (Fig. 4).

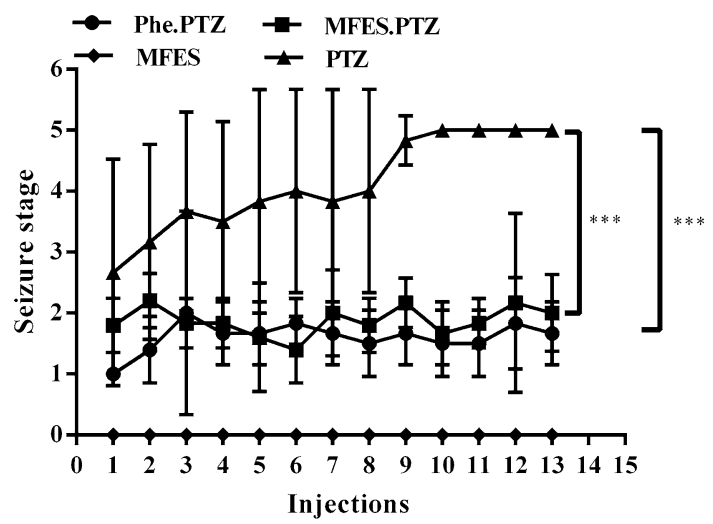

Fig. 1 Protective effect of MFES and phenytoin, on development of PTZ kindling. Statistical analysis showed that repeated administration of PTZ produced chemical kindling as indicated by the progression in seizure stage of PTZ-treated animals $[F(1,13)=419.71 ; p=0.001]$. Administration of phenytoin and MFES-inhibited the steady increase in seizure stage. $* * * p<0.001$, values are expressed as mean(SD)

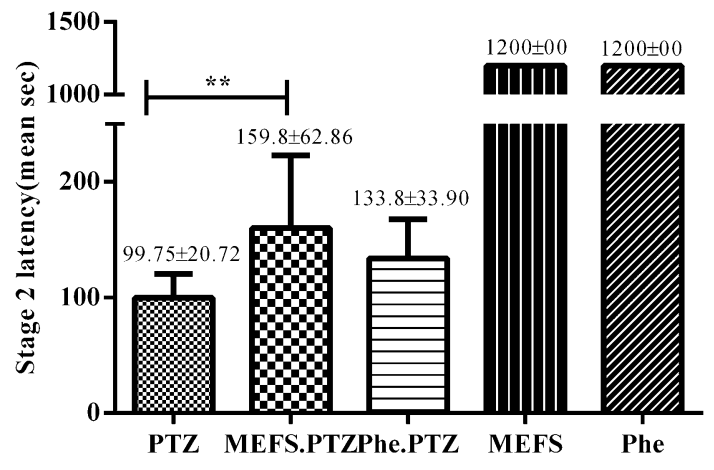

Fig. 2 Mild foot electrical stimulation increased stage two latency in PTZ kindled rats. One-way analysis of variance analysis showed significant differences among Phe.PTZ, MFES.PTZ and PTZ groups $[F(4,45)=3.75 ; * p<0.01]$, Post hoc analysis revealed that this difference was significant for MFES.PTZ group as compared to PTZ group $(p<0.010)$. Values are expressed as mean (SD)

\section{Effect of electrical stimulation on the development of PTZ kindling}

Electrical stimulation pretreatment significantly reduced the seizure stage for PTZ-treated animals $[p=0.001$, time effect $(F(12,286)=3.818, p<0.001)$, group effect $(F[1$, $286)=345.7, p<0.001)]$ and time-group interaction $(F(12$, $286)=3.278, p<0.001)$, and only one animal achieved stage five with 13 injections of PTZ (Fig. 1). Pretreatment with electrical stimulation significantly increased the time necessary to reach stage two of PTZ kindling $[F(4,45)=3.75$; $p=0.010$, one way ANOVA]. Post hoc analysis revealed that this difference was significant $(p=0.001)$ as compared to PTZ group (Fig. 2). Furthermore, stage five latency was significantly increased for animals in the electrical stimulation group compared to the PTZ group $[F(4,46)=31.07$;

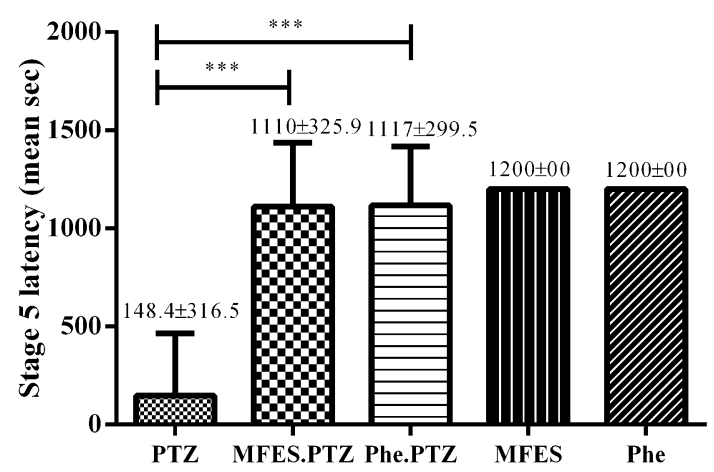

Fig. 3 Mild foot electrical stimulation has equal effect as phenytoin, on increasing the latency to stage five seizure. One-way analysis of variance analysis showed significant differences among PTZ, MFES. PTZ and phe.PTZ groups $[F(4,46)=31.07 ; * * * p<0.001]$, Post hoc analysis revealed that this difference was significant for PTZ.MFES group $(* * * p<0.001)$ and Phe.PTZ group $(p<0.001)$ as compared to PTZ group. Values are expressed as mean (SD) 


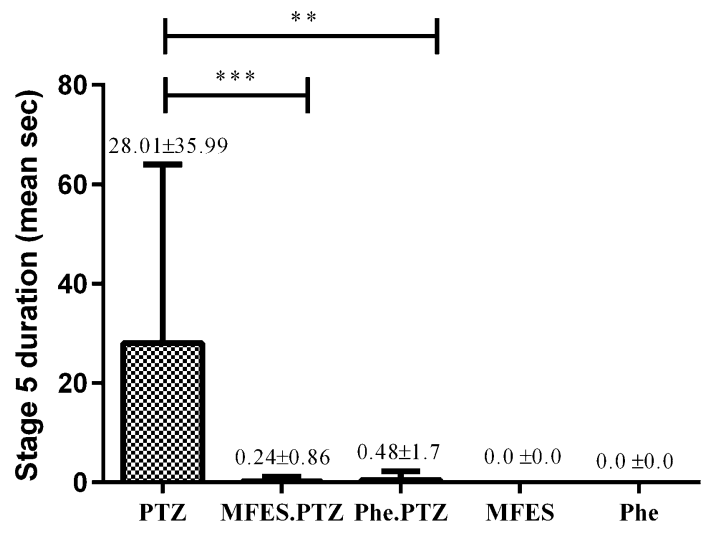

Fig. 4 Inhibitory effect of mild foot electrical stimulation and phenytoin, on the stage five duration. One-way analysis of variance analysis showed significant differences among PTZ, MFES.PTZ and phe.PTZ groups $[F(4,46)=5.501 ; p=0.001]$, Post hoc analysis revealed that this difference was significant for MFES.PTZ group $(* * * p=0.001)$ and phe.PTZ group $(* * p<0.01)$ as compared to PTZ group. Values are expressed as mean $(\mathrm{SD})$

$p=0.001$, one way ANOVA]. Post hoc analysis revealed that this difference was significant $(p=0.001)$ as compared to PTZ group (Fig. 3). Analyzing the effect of electrical stimulation on stage five duration also showed a significant reduction in duration $[F(4,46)=5.501 ; p=0.001$, one way ANOVA]. Post hoc analysis revealed that this difference was significant $(p=0.001)$ as compared to PTZ group (Fig. 4).

\section{Effect of electrical stimulation on established kindled rats}

The results showed that 10 days of MFES in rats with established kindled seizures had no effect on seizure parameters. When analyzing the effect of 26 days of MFES on PTZ kindled rats, results also showed that electrical stimulation after establishment of PTZ kindling had no effect on seizure parameters.

\section{Corticostrone test}

Comparing the plasma corticosterone concentrations in control, PTZ, phenytoin, MFES, MFES. PTZ and Phe.PTZtreated groups with one way ANOVA showed that there was no statistical difference in basal corticosterone levels among the groups $[F(5,30)=0.28, p=0.91]$.

\section{Discussion}

The results of present study show that applications of MFES every other day can retard the development of PTZ kindling in rats. This effect was comparable with phenytoin treatments $(30 \mathrm{mg} / \mathrm{kg})$. In addition, while electrical stimulation retarded development of kindling, it had no effect on established kindled seizures.

In this study, seizure severity consistently increased in rats treated with PTZ, which is in accordance with the results of earlier studies $[17,18]$.

Treatment with phenytoin elicited the expected inhibitory effect against PTZ-induced kindling. We have shown that the applied dose of phenytoin $(30 \mathrm{mg} / \mathrm{kg})$ partially inhibited the development of PTZ kindling. This result is consistent with previous reports $[19,20]$.

This is the first report demonstrating the antiepileptogenic effect of a MFES pretreatment on the PTZ model of epilepsy. Many experimental and clinical attempts have been made to control epileptic seizures using stressors such as electric shocks [21], immobilization [22], cold water [23] and acupuncture [24]. The main difference between this study and previous studies for stress induction was stimulus intensity. In the previous studies, high-intensity electrical stimulations ( $3 \mathrm{~mA}$ ) were used for electric foot stimulation. The intensity used in this study was $0.2 \mathrm{~mA}$, a much lower intensity than what has been used in previous research. Previous studies showed that an electrical stimulus with an intensity of $0.5 \mathrm{~mA}$ or higher increased corticosterone levels, while intensities lower than $0.5 \mathrm{~mA}$ did not increase the concentration of corticosterone [25]. In accordance with these findings, the results of corticosterone measurement have shown that electrical stimulation of the foot with $0.2 \mathrm{~mA}$ intensity did not change the concentration of stress hormone. This result is consistent with the finding of Uysal et al. [14] who observed no difference in corticosterone level with $0.2 \mathrm{~mA}$ foot electrical stimulation. So, it does not seem that the anticonvulsant effect of MFES was mediated by stress or stress hormones. Although no change in corticosterone concentrations was detected, we cannot rule out the potential role of stress. Previous studies have shown that MFES increased the level of BDNF and VEGF in the hippocampus, and hippocampus is an important structure in the development of kindling [26]. Therefore, the protective effect of MFES against PTZ kindling may result from the increase in the level of BDNF and VEGF in the hippocampus [14].

The exact nature of the anticonvulsant effect caused by electrical stimulation is not fully understood and requires further research.

After the first experiment showed that MFES could inhibit the development of PTZ kindling in rats, we investigated whether electrical stimulation could inhibit established kindled seizures or not. To answer this question, rats received MFES for 10 days after PTZ kindling. The results showed that the treatment did not change the seizure parameters. Another experiment was conducted in which rats received electrical stimulation for 26 days (same number as in the kindling development) after kindled seizures were 
fully established. The results showed that electrical stimulation for 26 days also did not change the seizure parameters. Perhaps it needs higher electrical current to inhibit established kindled seizures.

It seems that the kindling process was responsible for the different effects of MFES on development of kindling and on the established kindled seizures. Our data showed that MFES inhibited kindling development, but had no effect on established epileptic states. Previous studies have indicated that some conventional antiepileptic drugs can also cause independent effects for each of these phases. For example, Löscher et al. [27] have shown that phenytoin inhibits the development of amygdala kindling in non-kindled rats but that subsequent kindling of the animals leads to a total loss of the anticonvulsant efficacy of phenytoin. One interpretation of these results is that mild electrical stimulation blocked the underlying mechanism of kindling instead of masking the expression of seizures with anticonvulsant action.

Further studies are needed to shed light on the mechanism underlying the action of MFES on development of PTZ kindling. In addition, the potential ability of pure cutaneous somatosensory stimulation to prevent PTZ kindling requires further study. Because, molecular changes have not been investigated in this study we are not able to come out with a concise conclusion about the molecular mechanisms involved in anticonvulsant action of MEFS. Another limitation is that because the simultaneous electrophysiological recording of the brain area (such as amygdala and hippocampus) was not possible in this study. Therefore, the regions of the brain that are likely involved in anticonvulsant action of MFES were not determined.

In conclusion, our results showed that although MFES can suppress the development of PTZ kindling in rats, it had no effect on established kindled seizures.

Acknowledgments Financial support for this study was provided by Deputy Vice-chancellor of research on Arak University of Medical Sciences Grant \# 2283.

\section{Compliance with ethical standards}

Ethical approval Ethical approval for the study was provided by the Arak University of Medical Sciences Research Ethics Committee \# 94-132.

Conflict of interest The authors declare no conflict of interest.

\section{References}

1. Fiest K, Sauro K, Wiebe S et al (2017) Prevalence and incidence of epilepsy: a systematic review and meta-analysis of international studies. Neurology 88:96-303
2. Camfield C, Camfield P (2017) Cognitive disabilities and longterm outcomes in children with epilepsy: a tangled tail. Semin Pediatr Neurol 24(4):243-250

3. Mishra A, Goel RK (2014) Adjuvant anticholinesterase therapy for the management of epilepsy-induced memory deficit: a critical pre-clinical study. Basic Clin Pharmacol Toxicol 115(6):512-517

4. Dolton E, Choudry A (2014) Perampanel and challenging behaviour in intellectual disability and epilepsy: a management dilemma. Case Rep Psychiatry 2014:1-3

5. Robertson J, Hatton C, Emerson E, Baines S (2015) Prevalence of epilepsy among people with intellectual disabilities: a systematic review. Seizure 29:46-62

6. Mojs E, Gajewska E, Głowacka MD, Samborski W (2007) The prevalence of cognitive and emotional disturbances in epilepsy and its consequences for therapy. Ann Acad Med Stetin 53(3):82-87

7. Chen B, Choi H, Hirsch LJ, Katz A, Legge A, Buchsbaum R, Detyniecki K (2017) Psychiatric and behavioral side effects of antiepileptic drugs in adults with epilepsy. Epilepsy Behav 76:24-31

8. MacDonald B, Johnson A, Goodridge D, Cockerell O, Sander J, Shorvon S (2000) Factors predicting prognosis of epilepsy after presentation with seizures. Ann Neurol 48(6):833-841

9. Ito S (2016) GABA and glycine in the developing brain. J Physiol Sci 66:375-379

10. Stratton SC, Large CH, Cox B, Davies G, Hagan RM (2003) Effects of lamotrigine and levetiracetam on seizure development in a rat amygdala kindling model. Epilepsy Res 53(1):95-106

11. Gurbani S, Chayasirisobhon S, Cahan L, Choi S, Enos B, Hwang $\mathrm{J}$ et al (2016) Neuromodulation therapy with vagus nerve stimulation for intractable epilepsy: a 2-year efficacy analysis study in patients under 12 years of age. Epilepsy Res Treat 2016:9709056

12. Lehtimäki K, Möttönen T, Järventausta K, Katisko J, Tähtinen T, Haapasalo J et al (2016) Outcome based definition of the anterior thalamic deep brain stimulation target in refractory epilepsy. Brain Stimul 9(2):268-275

13. Jiao J, Jensen W, Harreby KR, Sevcencu C (2016) The effect of spinal cord stimulation on epileptic seizures. Neuromodul Tech Neural Interface 19(2):154-160

14. Uysal N, Sisman AR, Dayi A, Ozbal S, Cetin F, Baykara B et al (2012) Acute footshock-stress increases spatial learning-memory and correlates to increased hippocampal BDNF and VEGF and cell numbers in adolescent male and female rats. Neurosci Lett 514(2):141-146

15. Davoudi M, Shojaei A, Palizvan MR, Javan M, Mirnajafi-Zadeh J (2013) Comparison between standard protocol and a novel window protocol for induction of pentylenetetrazol kindled seizures in the rat. Epilepsy Res 106(1-2):54-63

16. Becker A, Grecksch G, Ruthrich H-L, Pohle W, Marx B, Matthies $\mathrm{H}$ (1992) Kindling and its consequences on learning in rats. Behav Neural Biol 57(1):37-43

17. Abasi-Moghadam M, Ghasemi-Dehno A, Sadegh M, Palizvan MR (2018) Improving effect of mild foot electrical stimulation on pentylenetetrazole-induced impairment of learning and memory. Epilepsy Behav 84:83-87

18. Tawfik KM, Moustafa YM, El-Azab MF (2018) Neuroprotective mechanisms of sildenafil and selenium in PTZ-kindling model: implications in epilepsy. Eur J Pharmacol 833:131-144

19. Staňková L, Kubová H, Mareš P (1992) Anticonvulsant action of lamotrigine during ontogenesis in rats. Epilepsy Res 13(1):17-22

20. Mishra A, Goel RK (2015) Comparative behavioral and neurochemical analysis of phenytoin and valproate treatment on epilepsy induced learning and memory deficit: search for add on therapy. Metab Brain Dis 30(4):951-958

21. Homayoun H, Khavandgar S, Dehpour AR (2002) The involvement of endogenous opioids and nitricoxidergic pathway in the 
anticonvulsant effects of foot-shock stress in mice. Epilepsy Res 49(2):131-142

22. Oliverio A, Castellano C, Puglisi-Allegra S (1983) Anticonvulsant effects of stress: role of endogenous opioids. Brain Res 271(1):193-195

23. Fournier NM, Galic MA, Kalynchuk LE, Persinger MA (2008) Profound hypothermia determines the anticonvulsant and neuroprotective effects of swim stress. Brain Res 1240:153-164

24. Kim ST, Doo AR, Kim SN, Kim SY, Kim YY, Kim JH (2012) Acupuncture suppresses kainic acid-induced neuronal death and inflammatory events in mouse hippocampus. J Physiol Sci 62:377-383

25. Shors TJ (2001) Acute stress rapidly and persistently enhances memory formation in the male rat. Neurobiol Learn Mem $75: 10-29$
26. Wittner L, Miles R (2007) Factors defining a pacemaker region for synchrony in the hippocampus. J Physiol 584(Pt 3):867-883

27. Löscher W, Cramer S, Ebert U (1998) Limbic epileptogenesis alters the anticonvulsant efficacy of phenytoin in Sprague-Dawley rats. Epilepsy Res 31(3):175-186

Publisher's Note Springer Nature remains neutral with regard to jurisdictional claims in published maps and institutional affiliations. 